\title{
Ultrafast Growth of Large Area Graphene on Si Wafer by a Single Pulse Current
}

\author{
Yifei Ge ${ }^{1,2}$, Mingming $\mathrm{Lu}^{1}{ }^{1}$, Jiahao Wang ${ }^{1}$, Jianxun $\mathrm{Xu}{ }^{1, *}$ and Yuliang Zhao ${ }^{1, *}$ \\ 1 Key Laboratory for Biomedical Effects of Nanomaterials and Nanosafety, CAS Center for Excellence in \\ Nanoscience, National Center for Nanoscience and Technology, Chinese Academy of Sciences, \\ Beijing 100190, China; geyf@nanoctr.cn (Y.G.); lumm2020@nanoctr.cn (M.L.); Wangih2020@nanoctr.cn (J.W.) \\ 2 School of Future Technology, University of Chinese Academy of Sciences, Beijing 100049, China \\ * Correspondence: xujx@nanoctr.cn (J.X.); zhaoyl@nanoctr.cn (Y.Z.)
}

check for updates

Citation: Ge, Y.; Lu, M.; Wang, J.; Xu J.; Zhao, Y. Ultrafast Growth of Large Area Graphene on Si Wafer by a Single Pulse Current. Molecules 2021, 26, 4940. https://doi.org/10.3390/ molecules 26164940

Academic Editors: Danilo Roccatano and Luca Valentini

Received: 22 June 2021

Accepted: 9 August 2021

Published: 15 August 2021

Publisher's Note: MDPI stays neutral with regard to jurisdictional claims in published maps and institutional affiliations.

Copyright: (C) 2021 by the authors Licensee MDPI, Basel, Switzerland. This article is an open access article distributed under the terms and conditions of the Creative Commons Attribution (CC BY) license (https:// creativecommons.org/licenses/by/ $4.0 /)$.

\begin{abstract}
Graphene has many excellent optical, electrical and mechanical properties due to its unique two-dimensional structure. High-efficiency preparation of large area graphene film is the key to achieve its industrial applications. In this paper, an ultrafast quenching method was firstly carried out to flow a single pulse current through the surface of a Si wafer with a size of $10 \mathrm{~mm} \times 10 \mathrm{~mm}$ for growing fully covered graphene film. The wafer surface was firstly coated with a 5-nm-thick carbon layer and then a 25-nm-thick nickel layer by magnetron sputtering. The optimum quenching conditions are a pulse current of $10 \mathrm{~A}$ and a pulse width of $2 \mathrm{~s}$. The thus-prepared few-layered graphene film was proved to cover the substrate fully, showing a high conductivity. Our method is simple and highly efficient and does not need any high-power equipment. It is not limited by the size of the heating facility due to its self-heating feature, providing the potential to scale up the size of the substrates easily. Furthermore, this method can be applied to a variety of dielectric substrates, such as glass and quartz.
\end{abstract}

Keywords: graphene; ultrafast growth; silicon

\section{Introduction}

Graphene, a two-dimensional honeycomb network crystal with monolayer atomic thickness, is favored by scientists all over the world for its excellent and unique optical, electrical, thermal, mechanical and other physical properties. Graphene can be used as an ideal material in fields such as multifunctional composites, organic optoelectronic materials, hydrogen storage materials, supercapacitors and microelectronic devices, electromagnetic attenuation materials and so on [1-4]. In 2004, single-layer graphene was first observed by Geim and Novoselov at Manchester University using the mechanical exfoliation method, which utilizes common cellophane tape to successively remove layers from a graphite flake. Despite its thermodynamic instability, graphene can exist stably in nature $[5,6]$ and this discovery rapidly caused researchers to develop several preparation methods and technologies over the past two decades. Among various synthesis methods, chemical vapor deposition (CVD) has proven to be the most popular and effective method to obtain large area, high-quality and layer-number-controllable graphene [7-10]. There are also some other novel preparation methods for graphene [11], such as the newly reported electrochemical exfoliation method [12,13]. Doped graphene is used for stochastic biosensors and can be easily obtained by electrochemically exfoliating graphite rods immersed in a solution without the need for high temperature.

Currently, people tend to obtain large area graphene in an economical synthesis method to realize its promising industrial applications. The CVD method usually uses a single crystal $\mathrm{Cu}, \mathrm{Ni}$ or alloy substrate, as well as polycrystalline metal substrate with specific crystal plane, to act as catalysts to promote the growth of graphene, then the grown graphene generally needs to be transferred to a target material. It is easy to cause defects 
such as wrinkles, breakages and contaminants in the transfer process, which greatly reduce the properties of the obtained graphene [14-17]. Moreover, it is difficult to obtain larger sized graphene by the CVD method due to the limitation of the furnace chamber. Another recently reported method, laser direct writing, has shown the potential to achieve large area or patterned graphene by the heating effect of laser. LinLi's team at Manchester University used laser irradiation to directly produce a large area graphene film with a size of about $3 \mathrm{~cm}^{2}$ in 3-6 s on a glass substrate pre-coated with a 5-28-nm-thick Ni film covered with olive oil [18]. However, a high-power laser source of $16 \mathrm{~kW}$ was required to give a laser spot of $60 \mathrm{~mm}$ with uniformly distributed energy. It is costly to use such a device for industrial production and is very difficult to scale up.

In general, the current growth methods for large area graphene are mainly restricted by the film transfer process, the size of furnace chamber and the high-power heat source for industrial applications. In our previous work, an ultrafast quenching method was reported to make graphene functional Ni tips using a pulse current [19]. In this work, we further utilized a single pulse current to flow through the surface of a Si substrate coated with 5-nm-thick $\mathrm{C}$ and 25 -nm-thick $\mathrm{Ni}$ for graphene growth. A large area and continuous few-layer graphene film was successfully generated on the surface of $S i$ substrate within $2 \mathrm{~s}$. As-grown graphene film proved to be highly conductive and covered the substrate fully.

\section{Results and Discussion}

The schematic diagram of the experimental set-up for ultrafast growth of large area graphene on $\mathrm{Si}$ wafer (300 $\mathrm{nm}$ of $\mathrm{SiO}_{2}$ on $\mathrm{Si}$ substrate) is shown in Figure 1a. The Joule heat produced by pulse current flew through the Ni coating on the surface of Si wafer was used to grow large area graphene at a high temperature instantly. As shown in Figure 1b, the Si wafer displayed dazzling white light during the heating process; Figure $1 \mathrm{c}$ is a wave of the optimal quenching conditions with a pulse current of $10 \mathrm{~A}$ and a pulse width of $2 \mathrm{~s}$ recorded by an oscilloscope. Figure $1 \mathrm{~d}$ is the photograph of the quenched $\mathrm{Si}$ wafers with a size of $10 \mathrm{~mm} \times 10 \mathrm{~mm}$. Figure 1e is the SEM images of graphene film; some dendritic nickel islands and holes were formed and the wrinkle of graphene film is clearly visible in the illustration. The Raman optical image in Figure 1f shows that the surface of the quenched Si wafers was relatively uniform. Nine points evenly distributed on the surface of the sample were selected for Raman testing with a $514 \mathrm{~nm}$ laser. The corresponding Raman spectra are in Figure $1 \mathrm{~g}$. We found a D peak near $1358 \mathrm{~cm}^{-1}$, the $G$ peak near $1581 \mathrm{~cm}^{-1}$ and the 2D peak near $2708 \mathrm{~cm}^{-1}$. Compared with the $\mathrm{G}$ and $2 \mathrm{D}$ peaks of single-layered graphene, the $G$ peak of as-grown graphene film shifts to a lower wavenumber, while the $2 \mathrm{D}$ peak shifts to a higher wavenumber, which prove that few-layered graphene has been grown [20]. At the same time, the ratio of $\mathrm{I}_{2 \mathrm{D}} / \mathrm{I}_{\mathrm{G}}$ is less than 1 , also implicating that the graphene produced was few-layered. The above results prove that few-layered graphene is almost fully covered on the surface of the sample. The quality of as-grown graphene, as well as the morphology of the surface of quenched Si wafer, was similar to that of graphene synthesized by laser direct writing [18].

The sheet resistance of the quenched $\mathrm{Si}$ wafer was measured by four-probe resistance tester (Res Map 178, CDE, Cleburne, TX, USA). The calculation formula is as follows:

$$
\mathrm{R}_{\square}=F \frac{\pi}{\ln 2} \frac{U}{I}=4.5324 \cdot F \frac{U}{I}
$$

$F$ is the correction coefficient. The $\mathrm{Si}$ wafer is square and the ratio of the sample lateral size to the probe spacing is 10; the correction coefficient is 0.927 . The measured and corrected values of five sets of points were $50.3167 \Omega \cdot \mathrm{sq}^{-1}, 44.4257 \Omega \cdot \mathrm{sq}^{-1}, 50.0836 \Omega \cdot \mathrm{sq}^{-1}$, $62.0398 \Omega \cdot \mathrm{sq}^{-1}, 62.751 \Omega \cdot \mathrm{sq}^{-1}$, respectively. These resistance values are more than one order smaller than those of the graphene glasses synthesized by CVD $\left(\sim 1000 \Omega \cdot \mathrm{sq}^{-1}\right)$ [21] and four times smaller than the graphene synthesized by the solid-phase laser direct writing $\left(\sim 205 \pm 19 \Omega \cdot \mathrm{sq}^{-1}\right)$ [22]. The average sheet resistance of the graphene film $\left(53.9234 \Omega \cdot \mathrm{sq}^{-1}\right)$ showed a good electrical conductivity of the graphene-functionalized Si substrate, indicat- 
ing the formation of large area and continuous graphene on Si. This agrees with the above Raman results.

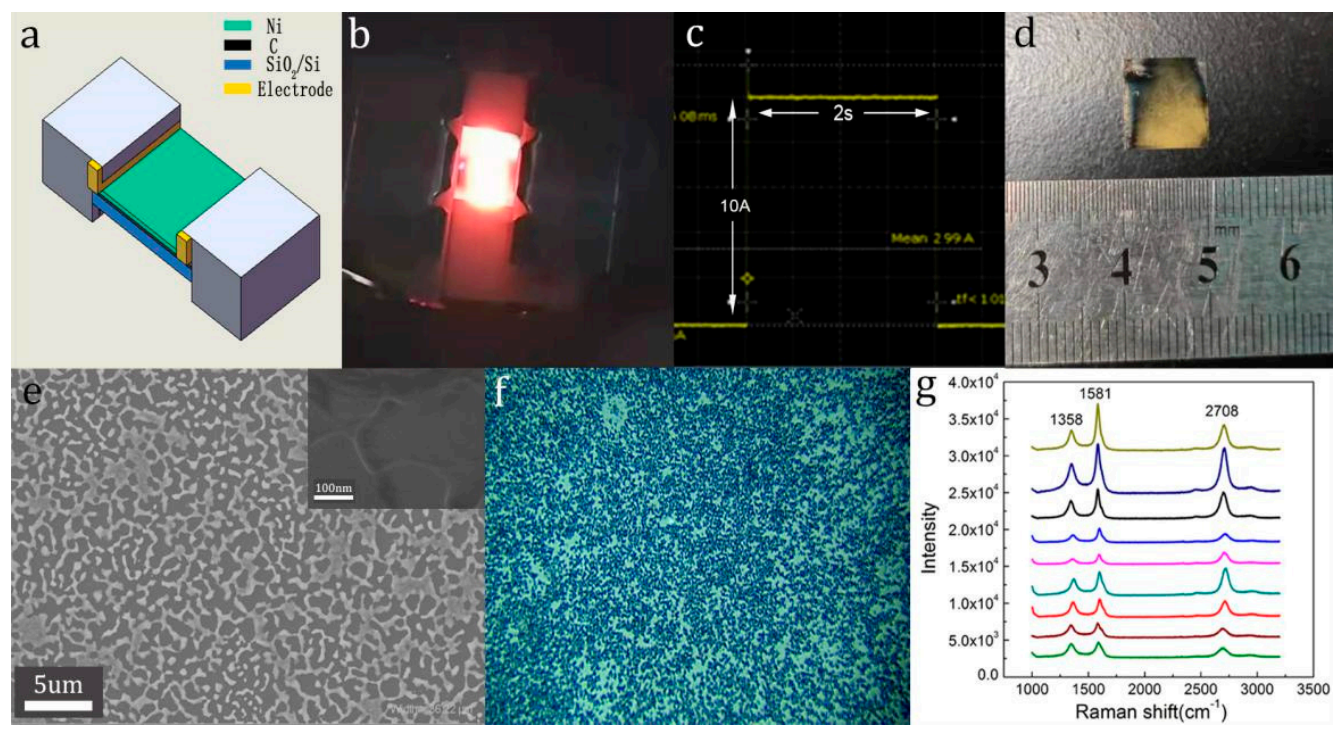

Figure 1. (a) Schematic diagram of experimental set-up for the growth of graphene film on the surface of Si wafer by ultrafast quenching method using pulse current; (b) photograph of Si wafer after a current flow through Cu electrode; (c) a single pulse of a current of $10 \mathrm{~A}$ with a pulse width of $2 \mathrm{~s}$, recorded by an oscilloscope; (d) photograph of graphene thin film on the surface of Si wafer; (e) high-resolution SEM(HR-SEM) image of the graphene, the wrinkles of graphene film are clearly visible in the illustration; (f) Raman optical diagram of graphene thin film in Figure 1d; (g) Raman spectra recorded at different positions showed the fully covered graphene film.

Our method is simple and highly efficient. The Joule heat produced by a pulse current within $2 \mathrm{~s}$ does not need any high-power equipment used for growth graphene. More importantly, it is not limited by the size of the heating facility due to its self-heating feature, providing the potential to scale up the size of the substrates easily.

Raman spectra were obtained by a spectroscope integrated with a SEM to further analyze in situ the corresponding relationship between the surface composition and the microstructure of the film on the surface of quenched Si wafer. The optical microscope image (Figure 2a), the SEM image (Figure 2b) and the Raman mapping (Figure 2c) of the same area of the graphene film were recorded. A Raman map labelled in red, blue and green areas is used to represent the distribution of the same type of Raman spectra with the corresponding colours in Figure 2d. By comparing the micro-morphology in Figure 2a-c, the Raman spectrum with blue colour corresponds to the dendritic islands on the quenched Si wafer surface and the Raman spectra with red and green colour correspond to the holes on the quenched Si wafer surface. For the Raman spectra with green colour, the value of $\mathrm{I}_{2 \mathrm{D}} / \mathrm{I}_{\mathrm{G}}$ is around 1 , which proves bilayer graphene has been grown in most areas of the holes. The value of $\mathrm{I}_{\mathrm{D}} / \mathrm{I}_{\mathrm{G}}$ in the blue Raman spectrum is obviously bigger than that in the red and green Raman spectra, suggesting that few-layer graphene on the surface of dendritic islands has more defects than the graphene on the holes.

The element composition and distribution of the graphene film on the surface of the quenched Si wafer are analyzed by the SEM elemental mapping in Figure 3a,b. The graphene film was mainly composed of four kinds of elements, namely $\mathrm{Ni}$, Si, O and $\mathrm{C}$. The dendritic islands, composed of $\mathrm{Ni}$ and $\mathrm{C}$ elements, can be identified as several hundred nanometer nickel-rich particles covered with graphene film, taking the according Raman spectra (Figure 2c,d) into account together. The holes are mainly composed of $\mathrm{Si}, \mathrm{C}$ and $\mathrm{O}$ elements, formed by thermal evaporation of $\mathrm{Ni}$ atom during heating process and the formation of few-layer wrinkled graphene film on the surface of Si substrates. Figure 3c,d show the $C 1$ s and Ni 2p XPS spectra of the as-grown graphene film. For the $C 1$ s region (Figure 3c), a peak at $285.3 \mathrm{eV}$ is attributable to the surface contaminant carbon. Another 
peak at $284.7 \mathrm{eV}$ can be assigned to the graphene. A peak at $285.7 \mathrm{eV}$ is attributable to the $\mathrm{Ni}_{3} \mathrm{C}$ and two peaks at $288.9 \mathrm{eV}$ and $286.6 \mathrm{eV}$ can be assigned to hydroxy and carbonyl functional groups attached to the graphene film. For the Ni 2 p region (Figure $3 \mathrm{~d}$ ), the Ni $2 \mathrm{p}_{3 / 2}$ peak is observed at $852.7 \mathrm{eV}$ and $853 \mathrm{eV}$; the binding energies agreed with those of the metallic fcc-Ni and hcp-Ni. A peak at $853.5 \mathrm{eV}$ is attributable to the $\mathrm{Ni}_{3} \mathrm{C}$. A peak at $855.7 \mathrm{eV}$ and a satellite peak at $861.3 \mathrm{eV}$ are attributable to the $\mathrm{NiO}$. We can conclude that the surface of the quenched wafer contains graphene, contaminant $\mathrm{C}, \mathrm{Ni}, \mathrm{Ni}_{3} \mathrm{C}$ and $\mathrm{NiO}$; the peaks are consistent with results reported in the literature [23-26].

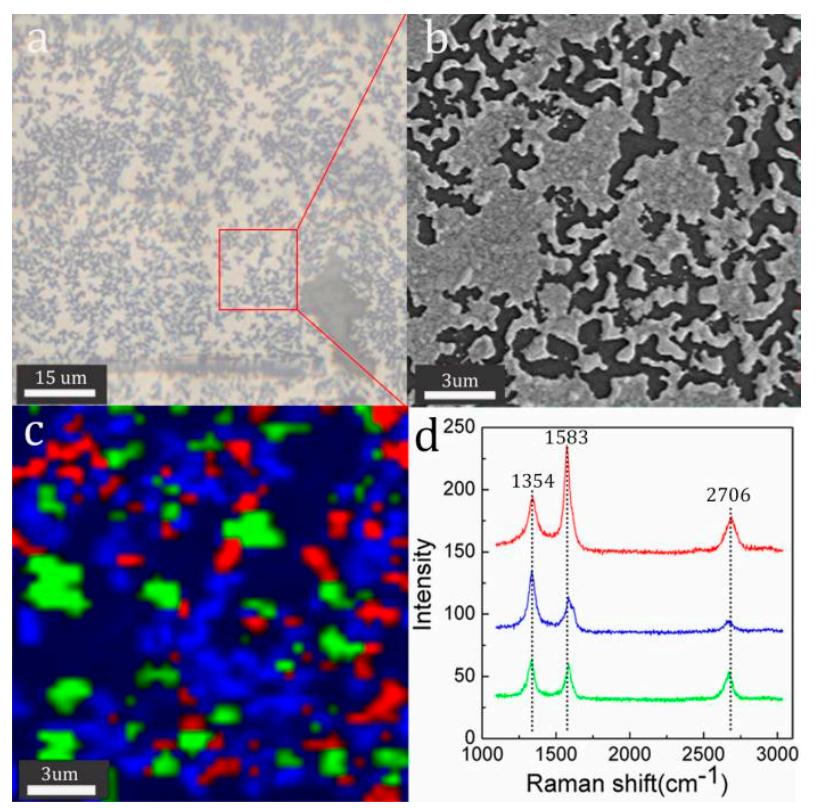

Figure 2. (a) Optical microscope image of the quenched Si wafer; the inserted image in Figure 2a is the corresponding selected area of SEM-Raman; $(\mathbf{b}, \mathbf{c})$ the SEM-Raman images $((\mathbf{b})$ is the corresponding SEM image and $\mathrm{c}$ is the corresponding Raman mapping); (d) three typical Raman spectra with different colours from the areas with corresponding colours in (c).

The corresponding AFM image of as-grown graphene film in Figure $3 e$ shows that many dendritic islands and holes were generated on the surface of the coating film during the annealing process, which corresponds to the above SEM image in Figure 3a. By using a white light interferometer, the morphology of a $113 \mathrm{um} \times 113 \mathrm{um}$ selected area is characterized in Figure 3f. The morphology was also very uniform, which indicates a large area of graphene film formed on the whole surface of the Si wafer.

Figure 4a shows Raman spectra of the graphene on Si wafer under different pulse currents. When the single pulse current increased from $6 \mathrm{~A}$ to $9 \mathrm{~A}$ with pulse width fixed at $2 \mathrm{~s}$, the value of $\mathrm{I}_{\mathrm{D}} / \mathrm{I}_{\mathrm{G}}$ decreased gradually, indicating a reduction of defects in the graphene. However, single-layer graphene was only generated at some points of the quenched $\mathrm{Si}$ wafer surface at the current form $6 \mathrm{~A}$ to $8 \mathrm{~A}$. At a current of $9 \mathrm{~A}$, the value of $\mathrm{I}_{2 \mathrm{D}} / \mathrm{I}_{\mathrm{G}}$ was around 1 and bilayer graphene with less defects were formed on nearly half of the quenched Si wafer surface. When the pulse current was increased to $10 \mathrm{~A}$, the Si wafer was covered with few-layered graphene, indicated by the value of $\mathrm{I}_{2 \mathrm{D}} / \mathrm{I}_{\mathrm{G}}$ smaller than 1. More importantly, the few-layered graphene film covered the full surface of Si wafer according to the results in Figure 1e,g. With increasing pulse width, the G peak shifted to a low wavenumber and the ratio of $\mathrm{I}_{2 \mathrm{D}} / \mathrm{I}_{\mathrm{G}}$ was gradually decreased, which proved that the layer of as-grown graphene changed from single to multiple. 

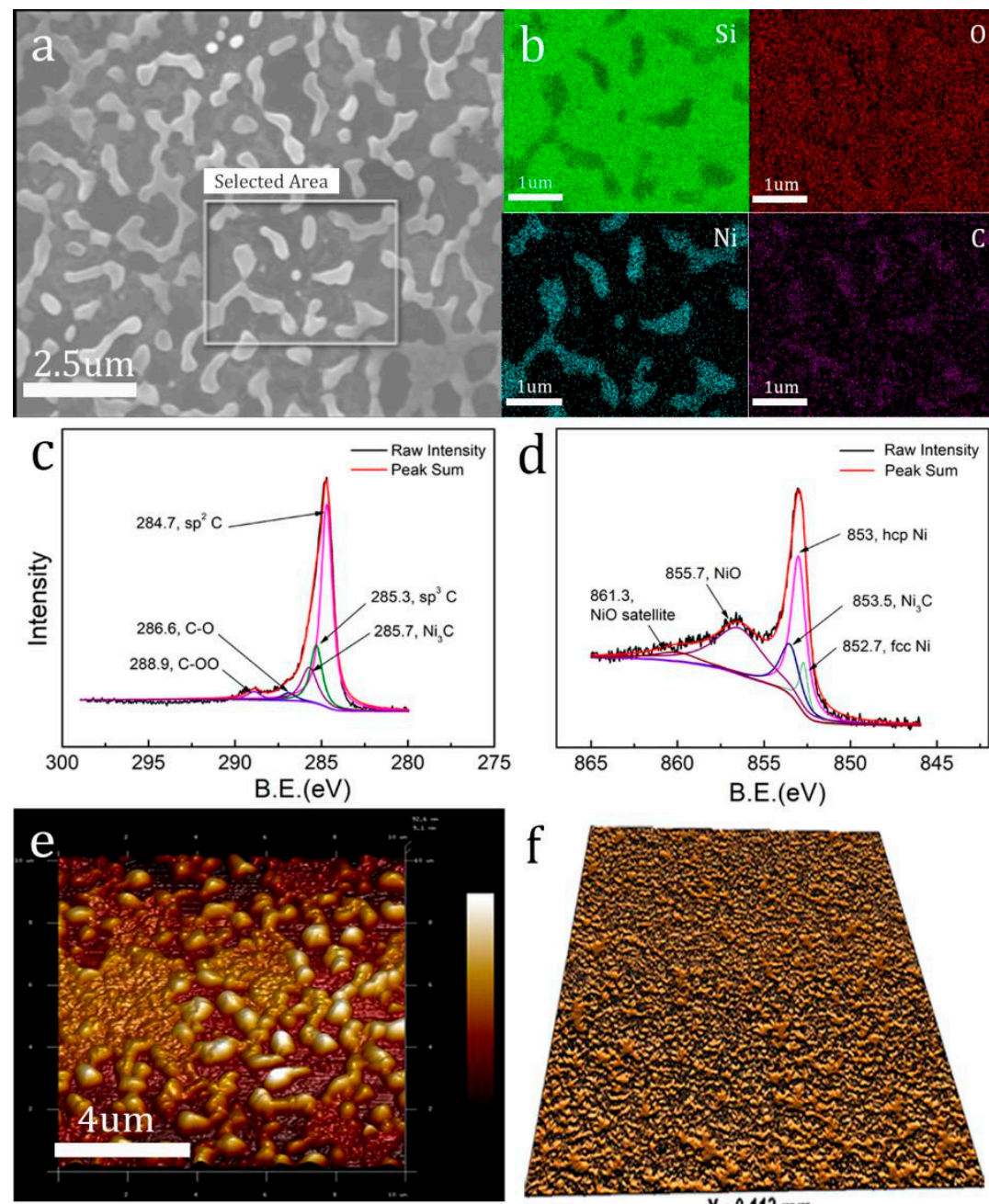

\section{$\mathrm{X}: 0.113 \mathrm{~mm}$}

Figure 3. (a) SEM image of as-grown graphene film on Si wafer surface; (b) the corresponding SEM elemental mapping image, scale bar is $1 \mathrm{um}$. Green, red, cyan and purple dots indicate $\mathrm{Si}, \mathrm{O}, \mathrm{Ni}$ and C atoms, respectively; (c,d) XPS spectra of C1s and Ni2p for as-grown graphene film on Si wafer surface; (e) AFM image of surface morphology of the as-grown graphene film with a size of $10 \mathrm{um} \times 10 \mathrm{um}$; (f) image of surface morphology of the as-grown graphene film with a size of $113 \mathrm{um} \times 113 \mathrm{um}$ by using white light interferometer.
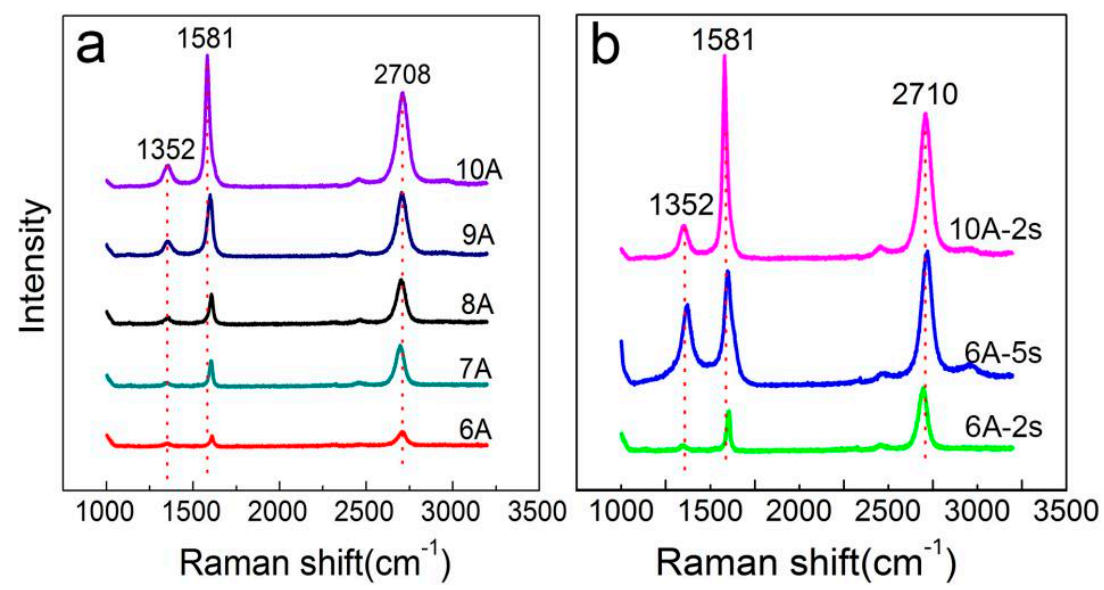

Figure 4. (a) Raman spectroscopy of the pulse current-dependent growth of graphene on Si wafer; (b) Raman spectroscopy of the pulse width-dependent growth of graphene on Si wafer. 
Figure $4 \mathrm{~b}$ shows the Raman spectra of the graphene on Si wafer under the different pulse width. At a pulse current of $6 \mathrm{~A}$, there is no obvious difference between the two types of Raman spectra with the increase of the pulse width from $2 \mathrm{~s}$ to $5 \mathrm{~s}$. A small pulse current results in a low surface temperature of the $\mathrm{Si}$ wafer, which is not enough to reach the growth temperature of large area graphene, even if the pulse width is $5 \mathrm{~s}$. At a pulse current of $10 \mathrm{~A}$, few-layered graphene was generated at the pulse width of $2 \mathrm{~s}$ while no graphene was generated at the pulse width of $5 \mathrm{~s}$ due to damage of the Si wafer at high temperatures. To summarize, the optimal annealing condition of a pulse current flowing through the surface of the $\mathrm{Si}$ wafer is $10 \mathrm{~A}$ and the pulse width is $2 \mathrm{~s}$.

The growth process of graphene film on the $\mathrm{Si}$ wafer by pulse current quenching could be revealed by comparing the microstructure and Raman spectra of the surface of the $\mathrm{Si}$ wafers before and after quenching under different pulse currents. Figure $5 \mathrm{a}$ shows the SEM image of the $\mathrm{Si}$ wafer successively coated with a 5-nm-thick C layer and a 25-nm-thick $\mathrm{Ni}$ layer before quenching; the surface is relatively flat. Figure $5 \mathrm{~b}$ and $\mathrm{c}$ are the SEM and HR-SEM images of the Si wafer after quenching with a pulse current of $6 \mathrm{~A}$ and a pulse width of $2 \mathrm{~s}$. Some light grey and black holes appear on the Ni layer in Figure 5b. Wrinkled graphene thin film was formed inside the holes in Figure $5 \mathrm{c}$, which was also proved by the Raman mapping results in Figure 5 d,e. The intensity of the Raman peaks of the green area was small, indicating that the graphene crystallinity was relatively weak on most of the surface of the Si wafer, where the morphology of the nickel layer was basically unchanged. Figure $5 \mathrm{f}$ is a HR-SEM image of the $\mathrm{Si}$ wafer after quenching with a pulse current of $10 \mathrm{~A}$ and a pulse width of $2 \mathrm{~s}$. A large area of dendritic nickel-rich islands was formed due to the spheroidization of the nickel layer at higher temperatures. Few-layered graphene covered the whole surface of the $\mathrm{Si}$ wafer.

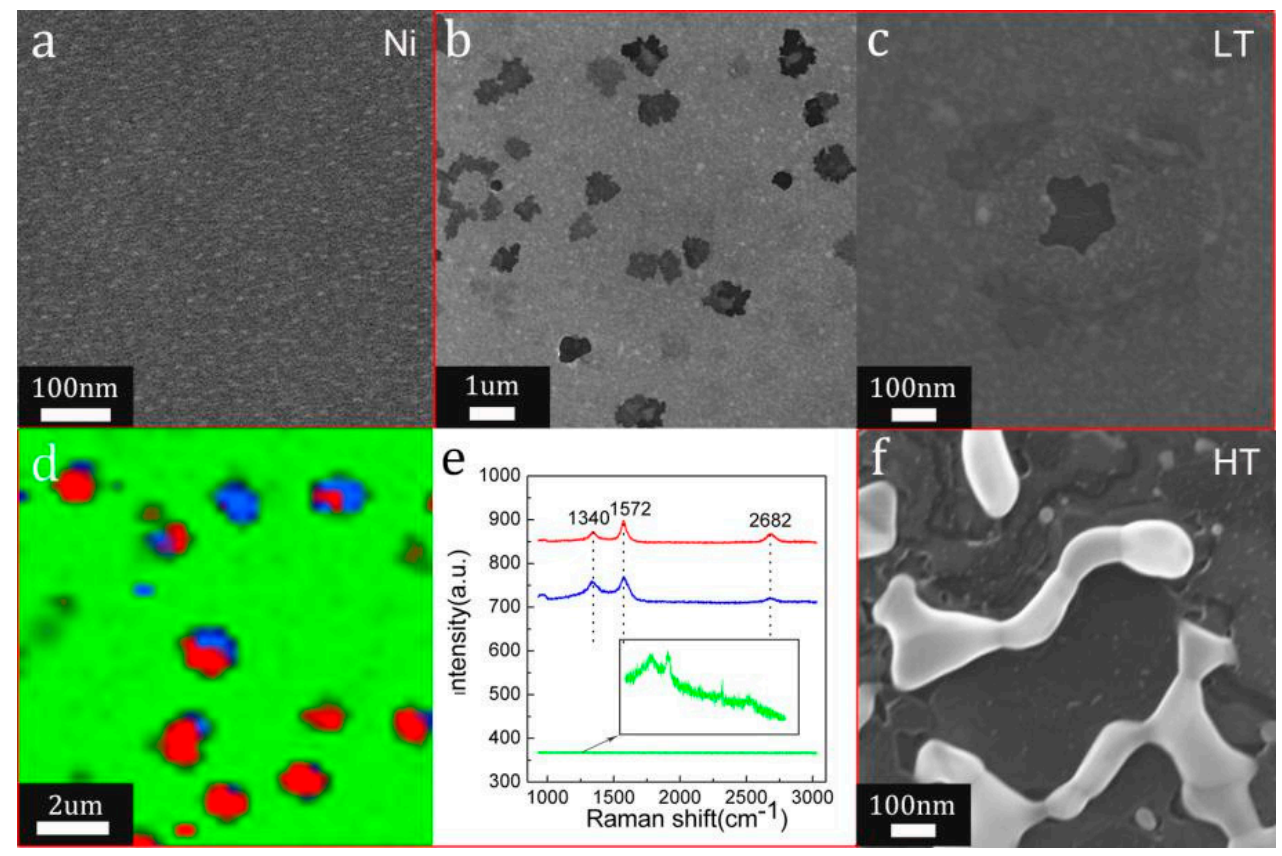

Figure 5. (a) SEM image of Si wafer coated with $\mathrm{C}$ and Ni layers before quenching; (b,c) the corresponding SEM and HR-SEM images after quenching with a pulse current of $6 \mathrm{~A}$ and a pulse width of 2 s; (d) Raman mapping of graphene; (e) Raman spectra of the red areas (red line), blue areas (blue line), green areas (green line) in (d); (f) HR-SEM image after quenching with a pulse current of $10 \mathrm{~A}$ and a pulse width of $2 \mathrm{~s}$.

Based on the above results, the growth process and mechanism of graphene film are discussed. In the heating process of quenching, a pulse current flew through the Ni layer on the $\mathrm{Si}$ wafer. Nickel carbide is firstly generated by utilizing the produced Joule heat, then decomposing into nickel atoms and carbon atoms at a temperature above $400{ }^{\circ} \mathrm{C}$; the nickel 
atoms evaporate and the carbon atoms are converted into graphene film at a temperature around $1100^{\circ} \mathrm{C}$ [27]. Some light grey and black holes firstly appear, then they are expanded with the increasing temperature. Some dendritic nickel-rich islands with a width of several hundreds of nanometers finally formed on the Si wafer after massive evaporation of nickel atoms. The islands were covered with few-layered graphene with a few defects and the holes were mostly covered with bilayer graphene with more defects. We proved the similar mechanism for graphene growth on Ni tip in our previous paper [19], which is different from the traditional segregation mechanism.

\section{Materials and Methods}

\subsection{Graphene Growth}

In our experiment, 5-nm-thick carbon thin film (the purity of carbon target is $99.99 \%$, Acs-4000-C4, ULVAC, Chigasaki, Japan) and 25-nm-thick nickel thin film (the purity of nickel target is $99.99 \%$, Lab-18, Lesker, Jefferson Hills, PA, USA) were formed successively on the $\mathrm{Si}$ wafers (300-nm-thick $\mathrm{SiO}_{2}$ on $\mathrm{Si}$ wafer, $10 \mathrm{~mm} \times 10 \mathrm{~mm} \times 0.4 \mathrm{~mm}$ thickness) by magnetron sputtering method.

The coated Si wafers were quenched by the following ultrafast quenching methods developed by us. As shown in the schematic diagram of the experimental set-up in Figure 1a, as a heating unit, a pair of copper electrode sheets with a length of $30 \mathrm{~mm}$, width of $1 \mathrm{~mm}$ and thickness of $0.05 \mathrm{~mm}$ were placed on both ends of surface of the coated Si wafer symmetrically. To ensure a good surface contact between the Si wafer and the copper electrode sheet, a ceramic block was placed on top of the electrode sheet, then the two ends of the ceramic block were fixed on the ceramic base with dovetail clips. The copper electrode sheets were connected to the external power supply through the vacuum electrode flange and the whole system was fixed in a vacuum chamber equipped with a molecular pump. After the vacuum reached $10^{-5} \mathrm{~Pa}$, a single pulse (generated by a HMP4030 DC electrical station, Rhodes and Schwartz company, Munich, Germany) with a current of $10 \mathrm{~A}$ and a pulse width of $2 \mathrm{~s}$ was provided by an external power supply for ultrafast heating. As shown in Figure 1b, the coated Si wafer displayed dazzling white light instantly during the pulse current flew through the copper electrode sheets; the corresponding current pulse waveform was recorded by an oscilloscope in the inserted image in Figure 1b. The whole quenching process was very instantaneous and the waveform showed that the elapsed time of the pulse current raised to $10 \mathrm{~A}$ was about $10 \mathrm{~ms}$ and a drop to $0 \mathrm{~A}$ was also recorded at the same time. During the heating process, the applied pulse current flew through the conductive coating on the surface of the Si substrate, which produced a large amount of Joule heat to make the surface of the Si substrate rapidly reach a red-hot state and then the Si wafer naturally cooled down. It was estimated that the heating rate was closer to $1000{ }^{\circ} \mathrm{C} / \mathrm{s}$ by calculation with the cooling rate.

\subsection{Materials Characterization}

Scanning electron microscopy (SEM, Hitachi S4800+EDS, Tokyo, Japan, 15 kV) studies were performed on as-grown graphene film on Si wafer surface to observe the surface morphologies and the corresponding element composition. Raman spectroscopy (Renishaw inVia plus, Wotton under Edge, UK, $514 \mathrm{~nm}$ excitation wavelength, $1 \mathrm{~mm}$ spatial resolution) was used to measure the characteristics of graphene. Surface topography images were obtained using an atomic force microscope (AFM, Bruker Multimode-8, Santa Barbara, CA, USA) and white light interferometer (BW-S501, Nikon, Tokyo, Japan). The graphene film was identified by using a RISE Raman system with a Sigma 300 SEM instrument (Carl Zeiss Microscopy Ltd., Jena, Germany). The laser wavelength was $532 \mathrm{~nm}$. The Raman mapping of graphene film was carried out with a grid spacing of $200 \mathrm{~nm}$ and an accumulation time of $1 \mathrm{~s}$ at each spot. Ni 2p and C 1s XPS spectra were recorded using an X-ray photoelectron spectroscopy (XPS, PHI5300, Perkin-Elmer, Fremont, CA, USA). $\mathrm{Al} \mathrm{K} \alpha$ radiation was used as an excitation source. The XPS analysis area was set to a 
diameter of $500 \mu \mathrm{m}$ and the step size to $0.05 \mathrm{eV}$ with a base pressure of 10-9 Pa during all measurements.

\section{Conclusions}

In this paper, we successfully grew a large area of graphene film on Si wafer using an ultrafast single pulse current quenching method. A single pulse current flew through the $\mathrm{Ni}$ layer on the surface of the Si wafer successively coated with a 5-nm-thick C layer and a 25-nm-thick Ni layer. During the process of quenching, nickel carbide was firstly generated at a lower temperature, then it decomposed into nickel atoms and carbon atoms with the temperature increasing (above $400^{\circ} \mathrm{C}$ ); the carbon atoms were converted into graphene at a higher temperature (around $1100^{\circ} \mathrm{C}$ ). The optimal quenching condition is a pulse current of $10 \mathrm{~A}$ and a pulse width of $2 \mathrm{~s}$. The thus-prepared few-layered graphene film on the Si wafers proved to cover the substrate fully with a size of $10 \mathrm{~mm} \times 10 \mathrm{~mm}$. Moreover, it showed a high conductivity with an average sheet resistance of about $53.9 \Omega \cdot \mathrm{sq}^{-1}$. Our method is simple and highly efficient and does not need any high-power equipment. The sample can be self-heated by the pulse current without the need for an additional heating facility to provide heat; this provides the potential to scale up the synthesized graphene film by adjusting the pulse current or pulse width applied to a larger size of different substrates. Furthermore, this transfer-free method can be applied to a variety of dielectric substrates, such as glass, quartz, sapphire and so on. The thus-produced graphene-functionalized various substrates can be used for sensors, electrical heating devices, biological scaffolds, and so on.

Author Contributions: Conceptualization, Y.G. and J.X.; Data curation, Y.G.; Formal analysis, Y.G., M.L., J.W. and J.X.; Funding acquisition, J.X. and Y.Z.; Investigation, Y.G.; Methodology, Y.G.; Supervision, J.X.; Visualization, Y.Z.; Writing—original draft, Y.G.; Writing—review \& editing, M.L. All authors have read and agreed to the published version of the manuscript.

Funding: This work was financially supported by the Ministry of Science and Technology of China (Grant No. 2016YFA 0203200).

Institutional Review Board Statement: Not applicable.

Informed Consent Statement: Not applicable.

Data Availability Statement: The data presented in this study are available in the article.

Conflicts of Interest: The authors declare no conflict of interest.

Sample Availability: Samples are available from the authors.

\section{References}

1. Wintterlin, J.; Bocquet, M.L. Graphene on metal surfaces. Surf. Sci. 2009, 603, 1841-1852. [CrossRef]

2. Dahal, A.; Batzill, M. Graphene-nickel interfaces: A review. Nanoscale 2014, 6, 2548-2562. [CrossRef]

3. Jacobson, P.; Stöger, B.; Garhofer, A.; Parkinson, G.; Schmid, M.; Caudillo, R.; Mittendorfer, F.; Redinger, J.; Diebold, U. Nickel Carbide as a Source of Grain Rotation in Epitaxial Graphene. ACS Nano 2012, 6, 3564-3572. [CrossRef]

4. Wen, B.; Cao, M.; Lu, M.; Cao, W.; Shi, H.; Liu, J.; Wang, X.; Jin, H.; Fang, X.; Wang, W.; et al. Reduced Graphene Oxides: LightWeight and High-Efficiency Electromagnetic Interference Shielding at Elevated Temperatures. Adv. Mater. 2014, 26, $3484-3489$. [CrossRef] [PubMed]

5. Novoselov, K.S.; Geim, A.K.; Morozov, S.V.; Jiang, D.; Zhang, Y.; Dubonos, S.V.; Grigorieva, I.V.; Firsov, A.A. Electric Field Effect in Atomically Thin Carbon Films. Science 2004, 306, 666-669. [CrossRef] [PubMed]

6. Novoselov, K.; Geim, A.K.; Morozov, S.; Jiang, D.; Katsnelson, M.I.; Grigorieva, I.V.; Dubonos, S.V.; Firsov, A.A. Two-dimensional gas of massless Dirac fermions in graphene. Nat. Cell Biol. 2005, 438, 197-200. [CrossRef]

7. Cabrero-Vilatela, A.; Weatherup, R.; Braeuninger-Weimer, P.; Caneva, S.; Hofmann, S. Towards a general growth model for graphene CVD on transition metal catalysts. Nanoscale 2016, 8, 2149-2158. [CrossRef] [PubMed]

8. Luo, J.; Wang, J. Direct growth of large area uniform bi-layer graphene films on silicon substrates by chemical vapor deposition. Mater. Res. Express 2019, 6, 095611. [CrossRef]

9. Huang, M.; Bakharev, P.V.; Wang, Z.-J.; Biswal, M.; Yang, Z.; Jin, S.; Wang, B.; Park, H.J.; Li, Y.; Qu, D.; et al. Large-area single-crystal AB-bilayer and ABA-trilayer graphene grown on a Cu/Ni(111) foil. Nat. Nanotechnol. 2020, 15, 289-295. [CrossRef] 
10. Hens, P.; Zakharov, A.A.; Iakimov, T.; Syväjärvi, M.; Yakimova, R. Large area buffer-free graphene on non-polar (0 0 1) cubic silicon carbide. Carbon 2014, 80, 823-829. [CrossRef]

11. Coros, M.; Pruneanu, S.; Staden, R.-I.S.-V. Review-Recent Progress in the Graphene-Based Electrochemical Sensors and Biosensors. J. Electrochem. Soc. 2020, 167, 037528. [CrossRef]

12. Ilie-Mihai, R.-M.; Staden, R.-I.S.-V.; Lungu-Moscalu, A.; Pogacean, F.; Pruneanu, S.M. Sulphur Doped Graphenes Based 3D-Needle Stochastic Sensors as New Tools for Biomedical Analysis. J. Electrochem. Soc. 2021, 168, 037509. [CrossRef]

13. Staden, R.-I.S.-V.; Gheorghe, D.-C.; Ilie-Mihai, R.-M.; Tudoran, L.-B.; Pruneanu, S.M. Stochastic biosensors based on N- and S-doped graphene for the enantioanalysis of aspartic acid in biological samples. RSC Adv. 2021, 11, 23301-23309. [CrossRef]

14. Shi, Q.; Tokarska, K.; Ta, H.Q.; Yang, X.; Liu, Y.; Ullah, S.; Liu, L.; Trzebicka, B.; Bachmatiuk, A.; Sun, J.; et al. Substrate Developments for the Chemical Vapor Deposition Synthesis of Graphene. Adv. Mater. Interfaces 2020, 7, 1902024. [CrossRef]

15. Reina, A.; Son, H.; Jiao, L.; Fan, B.; Dresselhaus, M.S.; Liu, Z.; Kong, J. Transferring and Identification of Single- and Few-Layer Graphene on Arbitrary Substrates. J. Phys. Chem. C 2008, 112, 17741-17744. [CrossRef]

16. Lin, L.; Peng, H.; Liu, Z. Synthesis challenges for graphene industry. Nat. Mater. 2019, 18, 520-524. [CrossRef]

17. Liang, X.; Sperling, B.A.; Calizo, I.; Cheng, G.; Hacker, C.; Zhang, Q.; Obeng, Y.; Yan, K.; Peng, H.; Li, Q.; et al. Toward Clean and Crackless Transfer of Graphene. ACS Nano 2011, 5, 9144-9153. [CrossRef] [PubMed]

18. Huang, Y.; Sepioni, M.; Whitehead, D.; Liu, Z.; Guo, W.; Zhong, X.; Gu, H.; Li, L. Rapid growth of large area graphene on glass from olive oil by laser irradiation. Nanotechnology 2020, 31, 245601. [CrossRef]

19. Ge, Y.; Xu, L.; Lu, X.; Xu, J.; Liang, J.; Zhao, Y. One Second Formation of Large Area Graphene on a Conical Tip Surface via Direct Transformation of Surface Carbide. Small 2018, 14. [CrossRef]

20. Ferrari, A.C.; Meyer, J.; Scardaci, V.; Casiraghi, C.; Lazzeri, M.; Mauri, F.; Piscanec, S.; Jiang, D.; Novoselov, K.; Roth, S.; et al. Raman Spectrum of Graphene and Graphene Layers. Phys. Rev. Lett. 2006, 97, 187401. [CrossRef]

21. Liu, B.; Wang, H.; Gu, W.; Zhou, L.; Chen, Z.; Nie, Y.; Tan, C.; Ci, H.; Wei, N.; Cui, L.; et al. Oxygen-assisted direct growth of large-domain and high-quality graphene on glass targeting advanced optical filter applications. Nano Res. 2020, 14, 260-267. [CrossRef]

22. Xiong, W.; Zhou, Y.S.; Hou, W.J.; Jiang, L.J.; Gao, Y.; Fan, L.S.; Jiang, L.; Silvain, J.-F.; Lu, Y.F. Direct writing of graphene patterns on insulating substrates under ambient conditions. Sci. Rep. 2014, 4, 4892. [CrossRef] [PubMed]

23. Kovács, G.; Bertóti, I.; Radnóczi, G. X-ray photoelectron spectroscopic study of magnetron sputtered carbon-nickel composite films. Thin Solid Film. 2008, 516, 7942-7946. [CrossRef]

24. Liu, X.; Liu, W.; Ko, M.; Park, M.; Kim, M.G.; Oh, P.; Chae, S.; Park, S.; Casimir, A.; Wu, G.; et al. Metal (Ni, Co)-Metal Ox-ides/Graphene Nanocomposites as Multifunctional Electrocatalysts. Adv. Funct. Mater. 2015, 25, 5799-5808. [CrossRef]

25. Furlan, A.; Lu, J.; Hultman, L.; Jansson, U.; Magnuson, M. Crystallization characteristics and chemical bonding properties of nickel carbide thin film nanocomposites. J. Phys. Condens. Matter 2014, 26, 415501. [CrossRef] [PubMed]

26. Goto, Y.; Taniguchi, K.; Omata, T.; Otsuka-Yao-Matsuo, S.; Ohashi, N.; Ueda, S.; Yoshikawa, H.; Yamashita, Y.; Oohashi, H.; Kobayashi, K. Formation of Ni3C Nanocrystals by Thermolysis of Nickel Acetylacetonate in Oleylamine: Characterization Using Hard X-ray Photoelectron Spectroscopy. Chem. Mater. 2008, 20, 4156-4160. [CrossRef]

27. Xiong, W.; Zhou, Y.S.; Jiang, L.J.; Sarkar, A.; Mahjouri-Samani, M.; Xie, Z.Q.; Gao, Y.; Ianno, N.J.; Jiang, L.; Lu, Y.F. Single-Step Formation of Graphene on Dielectric Surfaces. Adv. Mater. 2012, 25, 630-634. [CrossRef] 